\title{
RECHTSPRECHUNG
}

\section{Der Europäische Gerichtshof bestätigt die Sollbruchstellen der Anerkennung}

\author{
Anmerkung zum Urteil des EuGH vom 5. April 2016, verb. Rs. C-404/15 \\ und C-659/15 PPU (Aranyosi und Căldăraru)
}

\section{Von Michael Schwarz, Bogotá*}

1. Erlangt eine Justizbehörde, die einen Europäischen Haftbefehl vollstreckt, Informationen über das Vorliegen von Haftmängeln im Ausstellungsmitgliedstaat, ist sie verpflichtet zu prüfen, ob im konkreten Fall gesicherte Anhaltspunkte dafür vorliegen, dass die Person im Falle der Übergabe Gefahr läuft, unmenschlicher oder erniedrigender Behandlung im Sinne von Art. 4 der Charta der Grundrechte ausgesetzt $\mathrm{zu}$ werden.

2. Die vollstreckende Justizbehörde muss zu diesem Zweck die ausstellende Justizbehörde um zusätzliche Informationen bitten; Letztere ist verpflichtet, innerhalb der im Ersuchen gesetzten Frist Auskunft zu erteilen.

3. Bis zum Erhalt der erforderlichen Informationen muss die vollstreckende Behörde die Entscheidung aufschieben. Unter Sicherung der materiellen Voraussetzungen der Übergabe kann sie in Anbetracht der Art. 6 und 48 der Charta der Grundrechte die Inhaftierung zeitweilig aussetzen. Kann die Gefahr nicht innerhalb einer angemessenen Frist ausgeschlossen werden, entscheidet die vollstreckende Justizbehörde über die Beendigung des Übergabeverfahrens.

Urteil des EuGH vom 5. April 2016, verb. Rs. C-404/15 und C-659/15 PPU Leitsätze des Verfassers

\section{Vertrauen und Brüche}

Ist Misstrauen, wie Gandhi sagt, ${ }^{1}$ tatsächlich ein Zeichen von Schwäche? Wer, wie der EuGH, die Grammatik des Vertrauens bemühen will, um die Praktiken gegenseitiger Anerkennung in der Union zu beschreiben und $\mathrm{zu}$ rechtfertigen, ${ }^{2}$ kommt wohl nicht umhin, das vorgenannte Credo zu korrigieren. ${ }^{3}$ Ziel anerken-

* Dr. Michael Schwarz, LL.M. (NYU) ist Asistente Postdoctoral an der Universidad de los Andes, Facultad de Derecho. Der Verfasser dankt herzlich Liesa Plappert und Mattias Wendel für wertvolle Hinweise.

1 Vgl. R. Iyer, The Moral and Political Thought of Mahatma Gandhi, 2000, S. 313.

2 Rechtsprechungsnachweise in Fn. 7. Aus dem Schrifttum dazu C. Franzius, Recht und Politik in der transnationalen Konstellation, 2014, S. 276 ff.; A.-K. Kaufhold, Gegenseitiges Vertrauen - Wirksamkeitsbedingung und Rechtsprinzip der justiziellen Zusammenarbeit im Raum der Freiheit, der Sicherheit und des Rechts, EuR 2012, S. 408 ff.; E. Schmidt-Aßmann/G. Dimitropoulos, Vertrauen in und durch Recht, in: Weingardt (Hrsg.), Vertrauen in der Krise, 2011, S. $129 \mathrm{ff}$.

3 Kritisch K. Nicolaïdis, Trusting the Poles? Constructing Europe through mutual recognition, 14 Journal of European Public Policy (2007), S. 682, 688. 
nungsbasierter transnationaler Strafjustiz im Raum der Freiheit, der Sicherheit und des Rechts (RFSR) ist es, bestehende Jurisdiktionslücken zu schließen, um staatlichen Strafansprüchen zur Durchsetzung jenseits der eigenen Rechtsordnung zu verhelfen. ${ }^{4}$ Damit die horizontale Kooperation zwischen Justizbehörden nicht im gleichen Zug den Grundrechtsschutz aushebelt und die Betroffenen zu Opfern freiheitsbeschränkender Anerkennungspraktiken degradiert, ${ }^{5}$ bedarf es der Etablierung von Sollbruchstellen der Anerkennung in Gestalt grundrechtlicher Versagungsgründe. ${ }^{6}$ Gemeinhin sollen mitgliedstaatliche Behörden von der Grundrechtsredlichkeit ihrer Amtskolleg*innen ausgehen. Das nennt der EuGH seit geraumer Zeit schibbolethesk den Grundsatz gegenseitigen Vertrauens. ${ }^{7}$ Einen neuralgischen Punkt markierte bislang die „Abschöpfungsphase“". ${ }^{8}$ In dieser vierten Phase unionsrechtlicher Anerkennungsregimes zieht die ersuchende Stelle den Nutzen aus der Anerkennung: Wie vorliegend wird etwa die aufgrund eines Europäischen Haftbefehls überstellte Person im ersuchenden Mitgliedstaat einem strafrechtlichen Erkenntnisverfahren unterworfen oder eine gegen sie verhängte Strafe vollstreckt.

Wie aber ist zu verfahren, wenn sich Zweifel an der Vertrauenswürdigkeit des Gegenübers offenbaren? Die Antwort des Gerichtshofs in diesem jüngsten Akt der Haftbefehls-Saga ${ }^{9}$ fällt erwartungsgemäß und im Stile eines horizontalen Solange-Vorbehalts ${ }^{10}$ aus: Grundrechtsschutz sticht Anerkennungspflicht. Im Ergebnis jedenfalls. Um nicht die Anerkennungseuphorie, den föderativen Geist und guten Ruf der Anerkennung als ausgesprochen souveränitätsschonender ${ }^{11}$ aber wohl auch regulativ anspruchsvollsten ${ }^{12}$ Integrationsmethode $\mathrm{zu}$ beschädigen, setzt der Gerichtshof auf einen prozeduralen Puffer. Erst nachdem eine transnationale Rückversicherung die konkrete Gefahr der unmenschlichen und erniedrigen-

4 Vgl. etwa $V$. Mitsilegas, The Limits of Mutual Trust in Europe's Area of Freedom, Security and Justice: From Automatic Inter-State Coorporation to the Slow Emergence of the Individual, 31 Yearbook of European Law (2012), S. 319. Allgemein zum Ende der Übergangsperiode bei der strafjustiziellen Zusammenarbeit nach Lissabon F. Zeder, Justizielle Zusammenarbeit in Strafsachen nach dem Ende der Übergangsperiode: Normalität und Sonderfälle, EuR 2015, S. 487 ff.

5 Zum Paradigmenwechsel der Anerkennung im Raum der Freiheit, der Sicherheit und des Rechts etwa $C$. Janssens, The Principle of Mutual Recognition in EU Law, 2013, S. 257 ff.; M. Möstl, Preconditions and Limits of Mutual Recognition, 47 CMLRev (2010), S. 405, $408 \mathrm{ff}$.

6 Zum Begriff der grundrechtlichen Sollbruchstelle M. Schwarz, Grundlinien der Anerkennung im Raum der Freiheit, der Sicherheit und des Rechts, 2016, S. 5 f., 264 ff., $313 \mathrm{ff}$.

7 Seit EuGH, verb. Rs. C-187/01 und C-385/01 (Gözütok und Brügge), ECLI:EU:C:2003:87, Slg. 2003, I-1345, Rn. 33. Jüngst $V$. Mitsilegas, The Symbiotic Relationship Between Mutual Trust and Fundamental Rights in Europe's Area of Criminal Justice, 6 New Journal of European Criminal Law (2015), S. $457 \mathrm{ff}$.

8 Zum Phasenmodell unionsrechtlicher Anerkennungsregimes M. Schwarz, (Fn. 6), S. 265 ff., speziell zur Abschöpfungsphase S. 266 f., $299 \mathrm{ff}$.

9 Zur Haftbefehls-Saga und der Erosion der Grundrechte $A$. Albi, Erosion of Constitutional Rights in EU Law: A Call for ,Substantive Co-operative Constitutionalism', 9 Vienna Journal of Int. Const. Law (2015), S. 151 ff. und S. 291 ff. Zum Grundrechtsgefälle ferner S. Douglas-Scott, The EU's Area of Freedom, Security and Justice: a lack of fundamental rights, mutual trust and democracy?, 11 Cambridge Yearbook of European Law Studies (2008-09), S. 53, $73 \mathrm{ff}$.

10 Vgl. dazu I. Canor, Solange horizontal - Der Schutz der EU-Grundrechte zwischen Mitgliedstaaten, 73 ZaöRV (2013), S. 249, $269 \mathrm{ff}$.

11 Siehe etwa KOM (2000) 495 endg., S. 2; KOM (1999) 299 endg., S. 2.

12 K. Nicolaïdis (Fn. 3), S. 685. 
den Behandlung gemäß Art. 4 GRC aufgrund der Haftbedingungen im Ausstellungsmitgliedstaat bestätigt, darf der vollstreckende Staat das Verfahren aussetzen und ggf. beenden. Dadurch setzt der EuGH ein Ausrufezeichen für den transnationalen Grundrechtsschutz.

\section{Ausgangsverfahren und Vorlagefragen}

Stein des Anstoßes waren die identischen Vorlagefragen in ähnlich gelagerten Verfahren über die Vollstreckung Europäischer Haftbefehle. ${ }^{13}$ Gegen Herrn Aranyosi, einen in Bremerhaven lebenden ungarischen Staatsangehörigen, erließ der Ermittlungsrichter am Distriktgericht Miskolc in Ungarn (Miskolci járásbíróság) auf Grundlage des Tatverdachts wegen Einbruchsdiebstahls und Sachbeschädigung zwei Haftbefehle nach dem Rahmenbeschluss über den Europäischen Haftbefehl (im Folgenden: RBEUHB). ${ }^{14}$ Der rumänische Staatsangehörige Herr Căldăraru war in Rumänien wegen Fahrens ohne Fahrerlaubnis zu einer Gesamtfreiheitsstrafe von einem Jahr und acht Monaten verurteilt worden und wurde in Bremen aufgrund eines Europäischen Haftbefehls eines rumänischen Gerichts (Judecătoria Făgăraş) festgenommen. Unter dem Eindruck der teilweise prekären menschenrechtlichen Haftbedingungen in Ungarn und Rumänien kontaktierte die Generalstaatsanwaltschaft Bremen die ausländischen Behörden mit Blick auf mögliche Auslieferungshindernisse. In keinem der Fälle erhielt sie konkrete Auskunft zur vorgesehenen Haftanstalt. Dennoch wurden die Anträge auf Entscheidung über die Zulässigkeit der Übergabe an die ersuchenden Behörden beim zuständigen OLG Bremen gestellt ( $\$ 29$ Abs. 1 IRG). Dieses stellte einstweilen fest, dass die formalen Übergabevoraussetzungen nach dem IRG zwar vorlägen, hegte indes Zweifel an der Zulässigkeit der Übergabe angesichts $§ 73$ S. 2 IRG, wonach die Vollstreckungshilfe unzulässig ist, soweit sie im Widerspruch zu den in Art. 6 EUV enthaltenen Grundsätzen steht. Der EGMR hatte in jüngerer Vergangenheit für Ungarn und Rumänien wiederholt missbilligt, dass die Bedingungen der Unterbringung von Inhaftierten in überbelegten Haftzellen ohne ausreichende Beheizung und Warmwasserversorgung gegen Art. 3 EMRK (Art. 4, 52 Abs. 3 GRC) verstoße. ${ }^{15}$ Das OLG sah sich nicht in der Lage, über die Zulässigkeit der Auslieferungen gemäß $\S 73$ IRG und Art. 1 Abs. 3 RBEUHB zu entscheiden. Es legte dem Gerichtshof deshalb nach Art. 267 AEUV in beiden Verfahren sinngemäß

13 Zum Ausgangsverfahren siehe Rn. 28 bis 63 des hier besprochenen Urteils EuGH, verb. Rs. C-404/15 und C-659/15 PPU (Aranyosi und Căldăraru), ECLI:EU:C:2016:198.

14 Rahmenbeschluss 2002/584/JI des Rates vom 13. Juni 2002 über den Europäischen Haftbefehl und die Übergabeverfahren zwischen den Mitgliedstaaten (ABl. EG 2002 L 190/1) in der durch den Rahmenbeschluss 2009/299/JI des Rates vom 26. Februar 2009 (AB1. EU 2009 L 81/24) geänderten Fassung.

15 Zum Menschenwürdebezug M. Nussbaum, Hiding from Humanity, 2004, S. 90, 247 f. Völkerrechtlich $N$. Rodley/M. Pollard, The Treatment of Prisoners under International Law, 2011, S. $379 \mathrm{ff}$. Zur Inbezugnahme der Charta und rechtsvergleichenden Erwägungen durch den EuGH seit Lissabon G. de Búrca, After the EU Charter of Fundamental Rights: The Court of Justice as a Human Rights Adjudicator?, 20 Maastricht Journal of European and Comparative Law (2013), S. $168 \mathrm{ff}$. 
die Frage zur Vorabentscheidung vor, ob Art. 1 Abs. 3 RBEUHB so auszulegen sei, dass eine Auslieferung zum Zweck der Vollstreckung unzulässig ist, wenn gewichtige Anhaltspunkte dafür bestehen, dass die Haftbedingungen im Ausstellungsmitgliedstaat die Grundrechte der betroffenen Person verletzen oder aber der Vollstreckungsstaat in solchen Fällen die Auslieferungsentscheidung von einer Zusicherung der Einhaltung von Haftbedingungen abhängig machen kann oder muss. Mit der zweiten Vorlagefrage wollte das OLG wissen, ob Art. 5 und 6 RBEUHB so zu verstehen seien, dass die Ausstellungsbehörde selbst die notwendigen Auskünfte über die Vereinbarkeit der Haftbedingungen mit den grundrechtlichen Anforderungen erteilt oder insofern die nationale Zuständigkeitsordnung im ersuchenden Mitgliedstaat gelte.

\section{Entscheidung des EuGH}

$\mathrm{Zu}$ Beginn der Entscheidungsgründe rekapituliert der Gerichtshof die Funktion des Haftbefehlsregimes zur Schaffung eines paneuropäischen Raums der Freiheit, der Sicherheit und des Rechts und betont die systemtragende Kraft der einschlägigen Grundprinzipien. Als unabdingbar für das Gelingen jeglicher transnationaler Kooperation der Strafjustiz im Haftbefehlssystem nach dem Grundsatz der gegenseitigen Anerkennung erweise sich ein hohes Maß an Vertrauen zwischen den Mitgliedstaaten (Rn. 76 f. $)^{16}$. Gegenseitige Anerkennung und gegenseitiges Vertrauen gehen Hand in Hand.

In der Diktion des Gerichtshofs besagt der Grundsatz der gegenseitigen Anerkennung, dass alle Mitgliedstaaten untereinander verpflichtet sind, einem Europäischen Haftbefehl Folge zu leisten (Rn. 79). Die Verfahren wurden zu diesem Zweck von Generation zu Generation unionsrechtlicher Anerkennungsregimes stärker uniformiert, formalisiert und Versagungsgründe auf ein Mindestmaß reduziert. Jede Vollstreckungsverweigerung aus anderen als den sekundärrechtlich vorgesehenen Versagungsgründen ist unzulässig. Angesichts solcher Regelungen wie Art. 1 Abs. 3 RBEUHB sind die Mitgliedstaaten dabei angehalten, gemäß dem Grundsatz gegenseitigen Vertrauens davon auszugehen, dass alle übrigen Mitgliedstaaten das Unionsrecht und insbesondere die dort anerkannten Grundrechte beachten (Rn. 78).

Nach dieser Beteuerung der Leitidee gegenseitigen Vertrauens in die Rechtschaffenheit der übrigen Mitgliedstaaten konzediert der Gerichtshof, dass den Mitgliedstaaten gleichwohl kein blindes Vertrauen abverlangt würde (Rn.81-83). Insbesondere dann, wenn grundrechtliche Garantien betroffen sind, darf der Schleier des Vertrauens durchaus angehoben und näher hingesehen werden. Anerkennungsregimes der 1. Generation wie der RBEUHB kennen noch keine explizi- 
ten grundrechtlichen Versagungsgründe. ${ }^{17}$ In noch vagem Duktus spricht Art. 1 Abs. 3 RBEUHB davon, dass die Besorgung der Aufgaben nach dem Rahmenbeschluss nicht die Pflicht berührt, die Grundrechte und die allgemeinen Rechtsgrundsätze zu achten, wie sie in Artikel 6 EUV niedergelegt sind. ${ }^{18}$ Hinter dieser verhaltenen Formulierung verbirgt sich freilich nichts anderes als die Bekräftigung der Unionsgrundrechte als Sollbruchstellen sekundärrechtlicher Anerkennungsregimes (auch) für die Abschöpfungsphase.

Bei der Vollstreckung des Europäischen Haftbefehls handelt es sich um die Durchführung von Unionsrecht i.S.d. Art. 51 Abs. 1 GRC, sodass das Verbot unmenschlicher oder erniedrigender Strafe oder Behandlung nach Art. 4 GRC zur Anwendung gelangt. Über Art. 52 Abs. 3 GRC erfährt das Grundrecht, dem sowohl der inhärente Bezug zur Menschenwürde nach Art. 1 GRC $^{19}$ als auch Art. 15 Abs. 2 EMRK Unverbrüchlichkeit attestieren, einen nach oben offenen Gleichlauf mit Art. 3 EMRK (Rn. 84-87). Der EuGH folgert daraus, dass, soweit Anhaltspunkte dafür bestehen, dass einer betroffenen Person eine unmenschliche oder erniedrigende Behandlung während ihrer Inhaftierung im Ausstellungsmitgliedstaat droht, der prinzipielle Vertrauensvorschuss dahin und die Vollstreckungsbehörde verpflichtet ist, das „Vorliegen dieser Gefahr zu würdigen“ (Rn. 88). Zur Würdigung der „echten Gefahr“ darf sich die Behörde auf „objektive, zuverlässige, genaue und gebührend aktualisierte Angaben“ über die Haftbedingungen im Ausstellungsmitgliedstaat stützen, wie sie sich in Entscheidungen internationaler wie nationaler Gerichte oder Berichten von Organen des Europarats sowie aus dem System der Vereinten Nationen offenbaren (Rn. 89). Im Einklang mit Art. 3 EMRK besteht sogar eine positive Pflicht, sich der menschenwürdigen Haftbedingungen zu vergewissern (Rn. 90). Doch genügt das Vorliegen einer Gefahr ,für systemische oder allgemeine, bestimmte Personengruppen oder bestimmte Haftanstalten betreffende Mängel bei den Haftbedingungen“ für sich genommen noch nicht, die Vollstreckung des Haftbefehls zu versagen (Rn. 91-93); hierfür müssen die konkreten Umstände der bevorstehenden Inhaftierung die Annahme rechtfertigen, dass die betroffene Person Gefahr läuft, nach ihrer Übergabe an die ersuchenden Justizbehörden einer Verletzung des Art. 4 GRC ausgesetzt zu werden (Rn. 94). Die Vollstreckungsbehörde ist gem. Art. 15 Abs. 2 RBEUHB gehalten,

17 Neuere Anerkennungsregimes wie die Europäische Ermittlungsanordnung (EEA) beinhalten explizit (optionale) grundrechtliche Versagungsgründe, etwa für den Fall, dass ,,berechtigte Gründe für die Annahme bestehen, dass die Vollstreckung einer in der EEA angegebenen Ermittlungsmaßnahme mit den Verpflichtungen des Vollstreckungsstaats nach Artikel 6 EUV und der Charta unvereinbar wäre“, so Art. 11 Abs. 1 lit.f) RL 2014/41/EU des Parlaments und des Rates vom 3. April 2014.

18 Kritisch zu den teils erratischen grundrechtlichen Beschwörungsformeln C. Rijken, Re-Balancing Security and Justice: Protection of Fundamental Rights in Police and Judicial Cooperation in Criminal Matters, 47 CMLRev. (2010), S. 1455, 1475; V. Mitsilegas, The Constitutional Implications of Mutual Recognition in Criminal Matters in the EU, 43 CMLRev. (2006), S. 1277, 1292; S. Peers, Mutual Recognition and Criminal Law in the European Union: Has the Council got it wrong?, 41 CMLRev. (2004), S. 5, 17.

19 M. Schwarz, Die Menschenwürde als Ende der europäischen Wertegemeinschaft? Eine realistische Perspektive auf das Schutzdefizit nach Art. 1 der Grundrechtecharta, 50 Der Staat (2011), S. 533, 548 f., 551 mwN; dazu auch J. Waldron, Inhuman and Degrading Treatment: The Words Themselves, 23 Canadian Journal of Law and Jurisprudence (2010), S. $269 \mathrm{ff}$. 
weitere, für ihre einzelfallbezogene Gefahrenprognose notwendige Informationen bei der ersuchenden Behörde einzuholen und kann hierzu eine angemessene Frist setzen; die Ausstellungsbehörde ist wiederum zur Auskunftserteilung verpflichtet (Rn. 95-97).

Gelangt die Vollstreckungsbehörde aufgrund der bereits vorhandenen sowie der zusätzlich eingeholten Informationen zu dem Ergebnis, dass eine ,echte Gefahr“ besteht, so ist die Vollstreckung des Haftbefehls ,,aufzuschieben, aber nicht aufzuheben“ (Rn. 98) und die Vollstreckungsbehörde setzt Eurojust darüber in Kenntnis (Rn. 99). ${ }^{20}$ An die Aufschiebungsentscheidung schließt eine Verhältnismäßigkeitsprüfung, die Fortdauer der Haft betreffend an: Die Inhaftierung der betroffenen Person darf nach der Entscheidung nur fortdauern, solange die Dauer der Haft das nach Art. 6 GRC zulässige Maß nicht überschreitet und der Unschuldsvermutung gem. Art. 48 GRC Rechnung getragen wird (Rn. 100 f.). Als relevante Gesichtspunkte nennt der Gerichtshof etwa die Passivität der kooperierenden Behörden, Selbstverschulden der langen Verfahrensdauer, Straferwartung, Fluchtgefahr sowie die wesentliche Überschreitung der im Rahmenbeschluss bezeichneten Vollstreckungsfristen. ${ }^{21}$

Kommt die Vollstreckungsbehörde zu der Überzeugung, dass in Anbetracht der Gesamtumstände die vorläufige Freilassung anzuordnen ist, so hat sie durch fluchtverhindernde Maßnahmen zu gewährleisten, dass die materiellen Voraussetzungen für die Übergabe fortbestehen (Art. 12, 17 Abs. 5 RBEUHB) solange noch keine endgültige Entscheidung über die Nichtvollstreckung getroffen ist (Rn. 102). Kann die Vollstreckungsbehörde schließlich aufgrund der ihr zur Verfügung gestellten Informationen das Vorliegen einer echten Gefahr unmenschlicher oder erniedrigender Behandlung während der Inhaftierung im Ausstellungsmitgliedstaat innerhalb einer angemessenen Frist nicht ausschließen, hat sie über die Beendigung des Übergabeverfahrens zu entscheiden.

\section{Wirkungspfade und Würdigung}

Wir mögen die Entscheidung als Reaktion des Gerichtshofs auf die jüngsten Identitätsgebaren aus Karlsruhe lesen. ${ }^{22}$ Immerhin ging es in dem Fall, den das BVerfG im Dezember 2015 entschieden hatte, ${ }^{23}$ ebenfalls um die Anerkennungspflichten nach dem RBEUHB und ebenfalls hatte das BVerfG auf die Menschen-

20 Ergeht eine negative Gefahrenprognose zum wiederholten Mal gegen einen Mitgliedstaat, hat er diesen Umstand dem Rat mitzuteilen (vgl. Art. 17 Abs. 7 RBEUHB). Nach Art. 17 Abs. 3 RBEUHB beträgt die Regelfrist bis zu einer endgültigen Entscheidung über die Vollstreckung 60 Tage, soweit die betroffene Person der Übergabe nicht zustimmt.

21 Siehe EuGH, Rs. C-237/15 PPU (Lanigan), ECLI:EU:C:2015:474, Rn. 59 f.

22 In diese Richtung H. Sauer, Mit den eigenen Waffen geschlagen: Die Reaktion des EuGH auf den unbedingten Vorrang der Menschenwürde vor dem Unionsrecht nach dem BVerfG, Verfassungsblog, Beitrag vom 7. April 2016, http://verfassungsblog.de/mit-den-eigenen-waffen-geschlagen-die-reaktion-des-eugh-auf-denunbedingten-vorrang-der-menschenwuerde-vor-dem-unionsrecht-nach-dem-bverfg/.

23 BVerfG, Beschluss vom 15. Dezember 2015, 2 BvR 2735/14, ECLI:DE:BVerfG:2015:rs20151215.2bvr273514. Dem vom BVerfG zu beurteilenden Fall lag die Überstellung aufgrund einer in absentia-Verurtei- 
würde rekurriert, indem es Art. 1 und $20 \mathrm{GG}$ als Grund des strafrechtlichen Schuldprinzips und zugleich als Grenze der Vollstreckungspflicht auslotete, die eine Auslieferung nach Italien verbiete. Im Urteilsabspann heißt es einsilbig und nonchalant, einer Vorlage an den EuGH bedürfe es nicht, denn die richtige Anwendung des Unionsrechts sei derart offenkundig, dass für vernünftige Zweifel kein Raum bleibe. ${ }^{24}$ Die Vorlage an den EuGH zur allgemeinverbindlichen Klärung der vom BVerfG treffsicher aufgespürten Rechtsfragen vor dem Hintergrund des Art. 4a RBEUHB und Art. 1 GRC hätte Karlsruhe als Impulsgeber des europäischen Verfassungsgerichtsverbunds ${ }^{25}$ sicherlich besser zu Gesicht gestanden.

Abseits des Anerkennungsgerangels zwischen den beiden Spruchkörpern bietet sich an, die Entscheidung als konsequente Fortentwicklung eines Gedankens zu begreifen, den der Gerichtshof bei der Kalibrierung des mitgliedstaatlichen Selbsteintrittsrechts im Gemeinsamen Europäischen Asylsystem (GEAS) nach der damals geltenden Dublin-II-VO erstmals formuliert hatte. ${ }^{26}$

In der Rechtssache N.S. sah sich der EuGH verschiedenen Vorlagefragen gegenüber, die im Kern darauf abzielten, ob ein Mitgliedstaat aus Gründen mangelnden Grundrechtsschutzes von einem Selbsteintrittsrecht gegenüber dem eigentlich für das Asylverfahren zuständigen Mitgliedstaat Gebrauch machen könne. ${ }^{27}$ Der EuGH verwies zunächst auf den Grundsatz gegenseitigen Vertrauens, nach welchem die Vermutung gelten müsse, dass die Behandlung von Asylsuchenden in jedem Mitgliedstaat im Einklang mit der Grundrechtecharta sowie mit der Genfer Flüchtlingskonvention und der EMRK stehe. ${ }^{28}$ Bestehen jedoch ernstzunehmende Anhaltspunkte dafür, dass die Überstellung an einen anderen Mitgliedstaat zu einer Gefährdung von Grundrechtsverbürgungen führt - hier standen die Zustände in den Asyl- und Flüchtlingsunterkünften in Griechenland nach Art. 4 GRC und Art. 3 EMRK im Fokus - ist der Vertrauensvorschuss verspielt. Erforderlich seien in Abwägung mit den verfolgten Zielen der Verwirklichung des RFSR und konkret des GEAS ,systemische Mängel“ im zuständigen Mitgliedstaat, ${ }^{29}$ was der EuGH unter Verweis auf den EGMR ${ }^{30}$ für den Stand des Asylverfahrens in Griechenland bejahte. Sind den Behörden die systemischen Mängel bekannt oder müssten sie ihnen bekannt sein, ist von der Überstellung abzusehen und hat die

lung angesichts Art. 6 EMRK und dem strafrechtlichen Schuldprinzip zugrunde. Ausführliche Urteilsanmerkung T. Reinbacher/M. Wendel, i.E. EuGRZ 2016.

24 BVerfG (Fn. 23), Rn. 125.

25 Zum europäischen Verfassungsgerichtsverbund A. Voßkuhle, Der europäische Verfassungsgerichtsverbund, NVwZ 2010, S. 1 ff.; ders., „Integration durch Recht“ - Der Beitrag des Bundesverfassungsgerichts, JZ 2016, S. 161 ff. Zur Kooperation im Gerichtsverbund durch Zurückhaltung und Dialog auch F. Kirchhof, Kooperation zwischen nationalen und europäischen Gerichten, EuR 2014, S. 267, 272 f. Ferner K. Lenaerts, Kooperation und Spannung im Verhältnis von EuGH und nationalen Verfassungsgerichten, EuR 2015, S. 3 ff.

26 VO (EG) Nr. 343/2003 des Rates vom 18. Februar 2003.

27 EuGH, Rs. C-411/10 (N.S.), ECLI:EU:C:2011:865, Slg. 2011, I-13905.

28 EuGH (Fn. 27), Rn. 80.

29 EuGH (Fn. 27), Rn. 85 f.

30 EGMR (Große Kammer), Urteil vom 21. Januar 2011 - 30696/09 (M.S.S./Belgien und Griechenland), Ziff. 358, 360 und 367. 
Behörde die Zuständigkeitsprüfung fortzusetzen. ${ }^{31}$ Der Unionsgesetzgeber hat das Kriterium der systemischen Mängel unterdessen in Art. 3 Abs. 2 UAbs. 2 DublinIII-VO kodifiziert. Frei von Spannungen ist dieser Ansatz nicht, da nach dem EGMR das Vorliegen systemischer Mängel eine hinreichende, aber keine notwendige Bedingung ist. ${ }^{32}$

In Sachen Aranyosi u.a. befürworteten einige Mitgliedstaaten die Übertragung dieses Gedankens in casu. Anders Generalanwalt Bot, der die Sachverhaltsanalogie, die lediglich ihrer „Einfachheit“" wegen ,,verlockend“ anmute, ${ }^{33}$ mit fragwürdigen Argumenten ablehnt. Bereits der Wortlaut des 13. Erwägungsgrundes des RBEUHB schließe allein die Auslieferung - nicht aber die Übergabe! - bei drohender unmenschlicher oder erniedrigender Strafe oder Behandlung aus. ${ }^{34}$ Aus der fehlenden Erwähnung der „übergebenen“ Person, noch dazu in den Erwägungsgründen, zu schlussfolgern, der Unionsgesetzgeber habe dadurch die Übertragung des Grundsatzes ausschließen wollen, überzeugt nicht. Selbst wenn darin ein wie auch immer gearteter Wille des Gesetzgebers zu erkennen ist, steht dieser im Widerspruch zum autoritativen Text des Art. 1 Abs. 3 RBEUHB. Auch spricht der 12. Erwägungsgrund explizit davon, dass der Rahmenbeschluss die Grundrechte achtet und keinesfalls so zu verstehen ist, dass er untersagt, die Übergabe einer Person abzulehnen, wenn objektive Anhaltspunkte dafür vorliegen, dass diese z.B. aus Gründen des Geschlechts, der Rasse, Religion, ethnischen Herkunft, Staatsangehörigkeit, Sprache, politischen Überzeugung oder sexuellen Ausrichtung strafrechtlich verfolgt wird. Auch die Erwägung, Haftbefehlsregime und GEAS verfolgten unterschiedliche Zielsetzungen, ${ }^{35}$ geht ins Leere. Zum einen handelt es sich bei den zu übergebenden Personen vielfach um Tatverdächtige, also nicht ausschließlich um verurteilte Straftäter. Damit ist die Gegenüberstellung von strafrechtlich verfolgten Schuldigen im Gegensatz zu schutzsuchenden Personen im Rahmen des GEAS als rein rhetorisch entlarvt. Zum anderen gebührt selbstverständlich auch verurteilten Personen grundrechtlicher Schutz. Der unterschiedliche Harmonisierungsgrad als weiteres Unterscheidungsmerkmal betrifft die (vorgeschaltete) Frage der Anwendbarkeit der Unionsgrundrechte, die der

31 EuGH (Fn. 27), Rn. 94-98, 106-108. Klarstellend EuGH, Rs. C-4/11 (Puid), ECLI:EU:C:2013:740, Rn. 25-34.

32 Instruktiv $M$. Wendel, Menschenrechtliche Überstellungsverbote: Völkerrechtliche Grundlagen und verwaltungsrechtliche Konkretisierung, DVB1 2015, S. 731, 733 ff.; ders., Asylrechtlicher Selbsteintritt und Flüchtlingskrise - Zugleich ein Beitrag zu den Grenzen administrativer Entscheidungsspielräume im Mehrebenensystem, JZ 2016, S. 332, 335 ff.

33 GA Bot, Schlussanträge vom 3. März 2016 in den verb. Rs. C-404/15 und C-659/15 PPU (Aranyosi und Căldăraru), ECLI:EU:C:2016:140. Dessen Vorschlag lehnt eine uneingeschränkte Vollstreckungspflicht zwar im Ergebnis ebenso ab, doch sieht der GA diese Möglichkeit in einer allgemein primärrechtlichen (nicht: grundrechtsspezifischen) Verhältnismäßigkeitsprüfung begründet (Rn. 145 ff.), die in erster Linie dem Ausstellungsmitgliedstaat obliegt (vgl. insb. Rn. 154 f., 174). Dieses Vorgehen übergeht indes die spezifisch grundrechtliche Dimension der Haftbedingungen im Lichte des abwägungsresistenten Art. 4 GRC und entbehrt zudem eines operationablen Maßstabs für die Handhabung der Fälle, in denen die ausstellende Behörde ihrer Pflicht zur Wahrung der Verhältnismäßigkeit beim Erlass des Haftbefehls nicht nachgekommen ist, auch weil unklar bleibt, unter welchen Umständen die Vollstreckungsbehörde zur Wahrung der Verhältnismäßigkeit tätig werden kann.

34 Schlussanträge (Fn. 33), Rn. 46-48.

35 Schlussanträge (Fn. 33), Rn. 49-53. 
EuGH in beiden Fällen zutreffend positiv beantwortet hat. $\mathrm{Ob}$ die Inhaftnahme einer Person die Zweckbestimmung wie nach dem RBEUHB darstellt oder eine Ausnahmeerscheinung (GEAS), ${ }^{36}$ ändert gleichermaßen nichts an der Einschlägigkeit der Grundrechte immer dann, wenn es zur Inhaftierung kommt. Abgerundet wird das Analogieverbot mit zwei konsequentialistischen Überlegungen, die den Verdacht erhärten, dass die Argumentation vom Ergebnis geleitet ist. Erstens seien die ,praktischen Konsequenzen“ für das Haftbefehlsregime ,,von ganz anderer Tragweite“, denn mit der Übertragung des Grundsatzes aus dem Urteil N.S. wären die ,,vollstreckenden Justizbehörden verpflichtet, die Übergabe der gesuchten Person zu verweigern“'37. Es bestehe die offenkundige Gefahr, dass eine Straftat ungeahndet bliebe, was die Rechte und Freiheiten der übrigen Unionsbürger*innen beeinträchtigen würde. ${ }^{38}$

Dem ist mit Johan Callewaert, Vizekanzler der Großen Kammer am EGMR, zu entgegnen: Die Erwägungen müssen nicht etwa lauten ,Wie viel Grundrechte verträgt das System?“, sondern „Wie viel System vertragen die Grundrechte?" 39 Es gilt das Primat der Grundrechtstreue. Die Entgrenzung mitgliedstaatlicher Strafgewalt unter unionsrechtlichem Banner ist nicht für kleinere Münze zu bekommen. Nimmt man das Verbot unmenschlicher und erniedrigender Behandlung nach Art. 4 GRC als Ausfluss der unantastbaren Garantie menschlicher Würde gemäß Art. 1 GRC ernst, ${ }^{40}$ so besteht schlicht kein Raum für Abwägungen. Der folgende Hinweis des Generalanwalts, andernfalls ermuntere die Rechtslage tatverdächtige oder verurteilte Personen dazu, sich ins Ausland abzusetzen, um sich der heimischen Strafjustiz zu entziehen, gepaart mit dem Schreckensszenario neuer Zufluchtsstaaten, die hier wie da entstehen könnten, ${ }^{41}$ zeigt zwar Sensibilität für das Kernproblem der Debatte: Menschenunwürdige Haftbedingungen in einigen Mitgliedstaaten sind ein Problem für die gesamte Union. ${ }^{42}$ Dennoch sollten diese Missstände auf politischer Ebene zwischen den Mitgliedstaaten diskutiert und gelöst statt auf dem Rücken Einzelner ausgetragen werden.

Der EuGH tut gut daran, der Argumentationslinie des Generalanwalts nicht zu folgen. Indem die Große Kammer Art. 1 Abs. 3 des Rahmenbeschlusses dezidiert als Versagungsgrund kennzeichnet, bekräftigt sie die Grundrechte als Sollbruchstellen der Anerkennung und erteilt damit einer Entwicklung, die eine Verselbständigung und Hypostasierung der Grundsätze der gegenseitigen Anerkennung und des Vertrauens zulasten der Betroffenen forciert, eine klare Absage. Mit sei-

36 Vgl. Schlussanträge (Fn. 33), Rn. 53.

37 Schlussanträge (Fn. 33), Rn. 57 und später Rn. 82.

38 Schlussanträge (Fn. 33), Rn. 60.

39 J. Callewaert, Grundrechtsschutz und gegenseitige Anerkennung im Raum der Freiheit, der Sicherheit und des Rechts, ZEuS 2014, S. 79, 90; ähnlich BVerfGE 113, 273, 316 - Europäischer Haftbefehl.

40 M. Schwarz (Fn. 19), S. 548 f., 551.

41 Schlussanträge (Fn. 33), Rn. 63 f.

42 Zum Vorschlag, unionsrechtlichen Anerkennungsregimes zu diesem Zweck ein horizontales Konsolidierungsverfahren zu implantieren, siehe M. Schwarz (Fn. 6), S. 340 f. 
ner Entscheidung sendet der Gerichtshof ein Signal, dass man beim Grundrechtsschutz auf ihn vertrauen kann.

\section{"Das Flaggschiff der deutsch- sprachigen Kommentare"

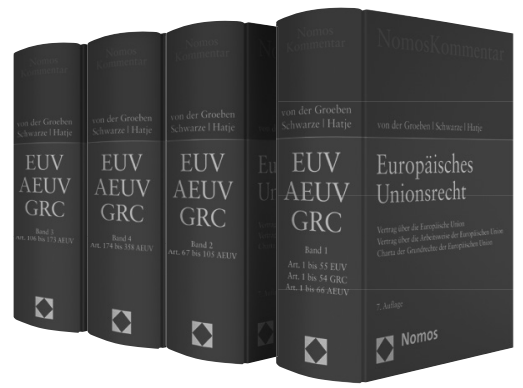

Europäisches Unionsrecht Vertrag über die Europäische Union - Vertrag über die Arbeitsweise der Europäischen Union - Charta der Grundrechte der Europäischen Union Herausgegeben von Dr. Hans von der Groeben, Prof. Dr. Dr. h.c. Jürgen Schwarze und Prof. Dr. Armin Hatje

7. Auflage 2015, 4 Bände, 8.372 S., geb., 890,-€ ISBN 978-3-8329-6019-3 www.nomos-shop.de/22204

Der Großkommentar ist das führende Standardwerk zum europäischen Recht. Seine wissenschaftliche Präzision und hohe Verständlichkeit ist meinungsprägend und integrationsfördernd. Seiner Argumentation folgen nationale und europä- ische Gerichte. Praxisnah wird die Sichtweise der europäischen Institutionen erläutert und hinterfragt. Auf hohem wissenschaftlichem Niveau fließt so die „Brüsseler" Sichtweise in die Meinungsbildung ein. 